\title{
Measurement and Evaluation Techniques to Estimate the Degradation Produced by the Radiated Transients Interference to the GSM System
}

\author{
Marc Pous, Marco A. Azpúrua, Member, IEEE, and Ferran Silva, Member, IEEE
}

\begin{abstract}
A common source of noise for digital communication systems is the radiated transient interference. As an example, it has been reported that the degradation is produced to the Global System for Mobile (GSM)-Railway signaling system when the impulsive noise is generated by sparks caused by the discontinuity between the catenary and the pantograph. Concerning the measurement and evaluation procedures defined in the electromagnetic compatibility standards, they are not suitable for these types of transient disturbances. Therefore, new techniques must be developed to determine, with a high confidence level, the degradation that impulsive noise will produce in communication systems. In this paper, novel time-domain measurement procedures are carried out to acquire transient interferences generated by sparks properly. Moreover, two different evaluation methodologies, employing base-band simulation and amplitude probability distribution detector, are used to analyze the impact of the disturbance applied into a GSM system interference scenario. Finally, the results obtained with the developed methodologies are successfully compared with a reference measure where the GSM downlink channel quality is monitored by a mobile station test unit.
\end{abstract}

Index Terms-Amplitude probability distribution (APD), electromagnetic transients interferences, GSM, impulsive noise, timedomain measurements.

\section{INTRODUCTION}

$\mathbf{I}$ $\mathrm{N}$ the electromagnetic compatibility (EMC) research area, the measurement and evaluation of the degradation produced by radiated transient disturbances on digital communication systems (DCSs) is a technological challenge that has not been completely addressed. The impulsive noise, which is generated by switching devices or by sparks, is a broadband interference that covers the spectrum from dc to several hundreds of megahertz or some gigahertz. Additionally, this man-made noise is characterized by its short and random burst parameters, which makes it really difficult to measure it correctly. Radiated transient interferences are propagated as an electromagnetic wave, reaching DCSs antennas and causing distortion

Manuscript received March 10, 2015; revised July 7, 2015; accepted August 8,2015 . This work was supported in part by EURAMET IND60EMC Research Project (the EMRP is jointly funded by the EMRP participating countries within EURAMET and the European Union) and by the Ministerio de Economía y Competitividad, under Project TEC2013-48414-C3-3-R.

The authors are with the Electromagnetic Compatibility Group, Universitat Politècnica de Catalunya, Barcelona 08034, Spain (e-mail: marc.pous@ upc.edu; marco.azpurua.a@ieee.org; ferran.silva@upc.edu).

Color versions of one or more of the figures in this paper are available online at http://ieeexplore.ieee.org.

Digital Object Identifier 10.1109/TEMC.2015.2472983 as well as system failures [1], [2]. For instance, previous research has found that the GSM-Railway signaling system is interfered by the impulse noise generated through the sparks produced by the discontinuity between the pantograph and the catenary [3], [4].

The EMC standards must prevent from interference situations where man-made noise produces failures of electronic products including the DCSs. However, electromagnetic transient interfering environments cannot be noticed beforehand if the measurements procedures described in the EMC standards are employed [5]. Radiated transient interferences are underestimated as most of EMC standards were developed to protect analog communication systems, which uses the signal-to-noise ratio as the main figure of merit. Moreover, in the standards, detectors such as the quasi-peak are still applied; nevertheless, it was developed to relate the human perception to the degradation of amplitude-modulated radio signals which are distant from the novel DCSs. Consequently, new measurement procedures must be considered to estimate, with a high level of confidence, the degradation produced to DCS [6], [7].

The main idea of the work presented in this paper is to demonstrate that it is possible to perform time-domain radiated emissions measurements of an interference environment and determine in advance the bit-error-rate (BER) produced in a DCS. To accomplish with this goal, time-domain measurements instead of frequency-sweep procedures defined in the EMC standards must be carried out obtaining the main parameters of the impulsive interferences. Furthermore, evaluation methodologies based on DCS baseband simulation and also the employment of statistical measurements are defined in this paper to deal with the radiated transient interferences.

Recently, statistical detectors have been introduced by CISPR, conversely, the usage of these detectors is not defined in any generic or product EMC standard. The amplitude probability distribution (APD) measurement offers the possibility to obtain the statistical parameters of the interferences, which is a really powerful tool to characterize the impulsive noise. Several studies have correlated APD measurements with the bit-error-probability (BEP) for coherent radio receivers [8], [9] and also novel time-domain APD measurement methodologies beyond EMC standards have been developed [10]. Furthermore, a method to define limits in the APD diagram in terms of the degradation produced to DCSs [11] has been published, thus providing the capability of evaluating the corresponding distortion produced by previously measured continuous interferences. 


\section{Proposed Methodologies}

As stated earlier, measuring radiated transient interferences is exceptionally challenging due to their random and broadband intrinsic characteristics. With the aim of protecting sensible communication receivers, measurements beyond EMC standards must be meticulously defined and carried out.

The following sections present novel acquisition and evaluation procedures developed to overcome the limitations of the EMC standards, making it feasible to obtain the time domain and the statistical information of the impulsive noise. Furthermore, combining transient interference measurements with methodologies for analyzing digital communications, we must be able to predict the BEP suffered by any DCS. Alternatively, the statistical information provided by the APD measurement can be related directly with the degradation of the communication link.

\section{A. IQ Capture and DCS Simulation Procedure}

This methodology is based on the in-phase and quadrature (IQ) components acquisition of the radiated interference in the frequency band of the communication system. Afterward, a simulation of the DCS adding the interference previously measured is performed to determine the degradation produced by the impulsive noise.

Concerning the setup of the time-domain measurement, in the transient acquisition, the receiver antenna is connected to the electromagnetic interference (EMI) receiver, which is centered in the working frequency of the DCS. The analog input stage of the EMI receiver is used to filter the transient interference in the communication frequency band and down converts it to the intermediate frequency (IF). The IF output of the EMI receiver is connected to the input of the oscilloscope, which performs the tasks to detect transient events employing the trigger and also to store the IF transient interference described by the following equation:

$$
x[n]=A_{\mathrm{IF}} \cdot \sin \left(2 \pi f_{\mathrm{IF}}(n)+\varphi_{\mathrm{IF}}\right),
$$

where $x[n]$ is the discrete input signal modulated in the $f_{\mathrm{IF}}$ frequency, where $n=k \Delta t$. As an example, the IF of the EMI receiver used in these measurements is $20.4 \mathrm{MHz}$. The IF signal is obtained, setting the most accurate resolution bandwidth (RBW) from the available ones in the EMI receiver. The RBW, which shall be selected by the user, must be larger than the bandwidth of the real time communication systems. Once the IF signal has been acquired, the measurement methodology is able to compute the IQ components of the interference employing the mathematical software. First, the data are bandpass filtered in the IF center frequency to avoid undesired spurious components. Second, the signal is demodulated obtaining the IQ components according to the following equations:

$$
\begin{aligned}
i[n] & =x[n] \cdot \cos \left(2 \pi f_{\mathrm{IF}}(n)\right) \\
q[n] & =x[n] \cdot \sin \left(2 \pi f_{\mathrm{IF}}(n)\right) .
\end{aligned}
$$

Afterward, $i[n]$ and $q[n]$ components of the interference are filtered with a low-pass finite impulse response filter equal to the RBW of the communication system. This is one of the main advantages that offer the measurement procedure, where the bandwidth can be equal to the bandwidth of the communication system. Therefore, the low-pass filter can be adjusted to obtain the portion of the interference that will be received by the DCS. The last stage of the postprocessing is to store the IQ data in a file; furthermore, in order to reduce the file size, the data are resampled with a lower sample rate. This sample reduction can be done because in the input of the postprocessing, we have the data modulated at $20.4 \mathrm{MHz}$, and at the end, the data are in baseband.

The second stage of the methodology is to employ the IQ baseband interference using it as the input for the base-band simulation of the communication system. The DCS simulation enables determining the BER of the communication system when the radiated transient interference is present in the environment. In order to estimate the performance of DCSs, computer-assisted techniques have been employed since the 70's [12]. The BER or BEP remains the most common figure of merit for a DCS. Computer simulations are often used to estimate the BEP in cases where the detection and/or decoding algorithms are too complex to admit useful mathematical expressions. If we consider that the transient interference channel also needs to be modeled, the bare mathematical approach becomes unmanageable due to the random characteristics in terms of amplitude, duration, frequency rate, and interval of the impulsive noise. The usual approach to overcome the none-model scenarios is to generate a sample of $N$ bits to simulate the processing required to transmit and detect these bits. Then, the number of erroneous bit decisions in the output can be counted and the BER is commonly estimated using the following equation:

$$
\mathrm{BER}=\frac{\text { Number of errors }}{N}
$$

where $N$ is the total number of bits transmitted.

As it has been explained, for a digital radio receiver, the BEP is used to evaluate the impact on the receiver performance when the disturbing noise is present in the channel. The BEP caused by a certain electromagnetic disturbance can be estimated and must be compared to the satisfactory requirements. These BEP requirements are strongly related with the service offered by the DCS and the quality of service defined in the standards. Examples of BEP typical requirements are $10^{-3}$ for speech communication and $10^{-6}$ for data transfer. Consequently, when the requirements are not fulfilled, a failure of the communication system is considered.

\section{B. APD Calculation and DCS Evaluation}

The second methodology, to determine if the radiated transient interferences generated by the sparks cause malfunction to the communication system, uses the APD diagram. The APD is defined as the amount of time that the measured envelope of an interfering signal exceeds a certain level [8]. Moreover, as it has been mentioned earlier, it is possible to establish limits in the APD diagram to analyze if certain interference will produce degradation on the DCS. Therefore, using the APD data obtained from the measurement and calculating the limits referred 
to the technical specification, we will be able to estimate the degradation produced by impulsive noise sources.

The APD gives information about the envelope statistics from the IF filter output, which corresponds to the required information for performance evaluation in the radio receiver. Therefore, it is necessary to carry out an envelope detection using the interference filtered by the RBW in the IF frequency band if the RBW is equal to the DCS channel bandwidth. In this situation, the envelope detection is reached by means of the Hilbert Transform, which is used extensively for analysis and signal processing in passband communication systems. Otherwise, the envelope of the radiated transient interference can be obtained from the IQ components obtained in the postprocessing stage as

$$
r[n]=\sqrt{i[n]^{2}+q[n]^{2}} .
$$

and the relation between $\mathrm{APD}_{R}(r)$ and the probability density function (pdf) of the envelope $R$ is

$$
\operatorname{APD}_{R}(r)=1-F_{R}(r)
$$

and

$$
f_{R}(r)=\frac{d}{d r} F_{R}(r)=-\frac{d}{d r} \operatorname{APD}_{R}(r)
$$

where $F_{R}(r)$ is the cumulative distribution function (cdf) and $f_{R}(r)$ is the pdf. The cdf describes the probability that a value of the envelope variable $R$ with a given pdf will be found to have a value less than or equal to $r$.

Finally, the APD is directly obtained from the cdf using the expressions shown above. The APD is plotted with the percentage of time, the ordinate is exceeded on the $y$-axis and the envelope values on the $x$-axis. These values are in accordance with the APD measurement function proposed by CISPR to make use of the correlation between the BEP and the APD. In the APD results presented in this paper, plot units of the envelope ( $x$-axis) have been set to power units referenced to 1 milliwatt $(\mathrm{dBm})$. However, they can be converted to voltage values or electric field applying the corresponding antenna factor.

Regarding the limits that must be introduced to the APD diagram, in general terms, those limits dots are related to the probability required by the system specifications and also to the amplitude of the received signal. Consequently, we will be able to compute and plot the APD limit points in the APD diagram following the equation described below when the shape of the APD diagram has a horizontal plateau [11]:

$$
\left(u_{\text {limit }}, P_{\text {limit }}\right) \equiv\left(\frac{\beta A}{\sqrt{m}}, P_{\text {req }}\right)
$$

where $A$ is the rms amplitude of the communication signal, $P_{\text {req }}$ is the probability required by the communication system, $m$ is the number of bits transmitted by one symbol, and $\beta$ is defined as

$$
\beta \equiv \frac{d_{\min }}{2} \frac{1}{\sqrt{E_{b}}}
$$

where $d_{\min }$ is the minimum distance of the constellation diagram and $E_{b}$ denotes the bit energy.
TABLE I

GSM-R SYSTEM SPECIFICATIONS

\begin{tabular}{lc}
\hline \hline Parameter & Value \\
\hline Downlink frequency band & $921-925 \mathrm{MHz}$ \\
Channel separation & $200 \mathrm{kHz}$ \\
Received power (P) & $-100 \mathrm{dBm} \leq \mathrm{P} \leq-40 \mathrm{dBm}$ \\
Bit rate & Up to $14.4 \mathrm{kbits} / \mathrm{s}$ \\
Modulation & GMSK (BT 0.3) \\
\hline \hline
\end{tabular}

\section{TEST SCENARIO}

\section{A. GSM System Interfered by Radiated Transients Produced by Sparks}

In this paper, the GSM system will be interfered by radiated transients generated by sparks. The purpose of choosing this communication system is to reproduce a common interference scenario in the laboratory. The goal is to emulate when the GSM-R system is interfered by the arching effect caused by the discontinuity between the pantograph and the catenary [13]. The GSM-R antenna located at the roof of the rolling stock receives the signal coming far away from the base transceiver station (BTS) and also the close radiated transient interference. In the GSM-R system, the received power of the downlink channel is between -40 and $-100 \mathrm{dBm}$, therefore, the electrical discharge produced by the air breakdown generates a transient interference strong enough to disturb the GSM-R downlink, which is located between 921 and $925 \mathrm{MHz}$. When the downlink is interfered, the communication link can be lost and the rolling stock goes toward a safe mode reducing the speed or stopping the train. In Table I, a summary of the GSM-R specifications is shown.

\section{B. Laboratory GSM Interference Scenario}

The interfering scenario that has been built in the test laboratory to interfere and measure the disturbance produced to the GSM system with the different methodologies is described as follows. For the sake of the experimental assessment, the aforementioned transient interference phenomenon was reproduced by following setup: first, the test has been performed in an anechoic chamber avoiding uncontrolled interferences. Inside the anechoic chamber, the Schlöder electrostatic discharge (ESD) simulator model SESD 200 in conformity with EN 61000-4-2 was used to generate the sparks to interfere the GSM system. The ESD gun emulates the sparks that are produced by the discontinuity between the pantograph and the catenary in the railway. In [14], the main parameters of the interference produced by the sparks of a real catenary were identified, establishing that the mean rise time is $0.4 \mathrm{~ns}$ and the duration is $5 \mathrm{~ns}$, which are similar to the interference defined in IEC 61000-4-2. The sparks generated by the ESD gun in the laboratory produce an electromagnetic field that reaches a mobile station (MS) and also a GSM antenna, both are placed close to the spark generator at $50 \mathrm{~cm}$.

Concerning the developed methodologies used to evaluate the performance of the GSM system when it is interfered by the sparks, the measurement system includes a GSM antenna, an 


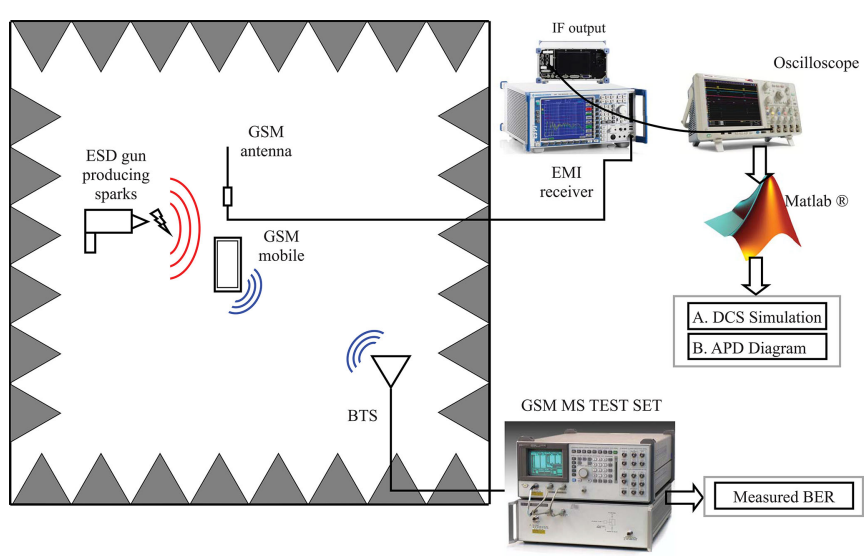

Fig. 1. Measurement setup of the GSM interfered system.

EMI receiver, and an oscilloscope. The GSM antenna is directly connected to the EMI receiver and the IF output is directly connected to the oscilloscope input. The IF data are captured in the time domain to afterward, by means of postprocessing employing MATLAB, to obtain the IQ components of the sparks interference in the frequency band of the GSM downlink channel. The GSM system is simulated employing Simulink, and the previous IQ measurement is added to the DCS simulation. The output of the simulation will allow us to estimate the BER of the communication system in the presence of the intentionally generated sparks produced by the ESD gun.

Alternatively, to obtain the results of the APD methodology, a different postprocessing is employed to reach the APD diagram employing the same time-domain measurements used earlier. In the next sections, a more detailed description is provided for each methodology. In Fig. 1, a schematic of the measurement and evaluation techniques described in this section is shown.

Regarding the sparks generated to interfere the GSM system, they were generated placing a metallic slice close to the air-discharge test electrode. The repetition frequency of the discharge pulses depends on the distance between the discharge electrodes and the metal where the discharge is produced. With the aim to study several cases of interference, the distance was varied to produce different repetition rate of the sparks. The first distance selected is the minimum distance required to trigger the spark. This distance will allow evaluating the case with the highest repetition rate. Moreover, a larger distance equal to $1 \mathrm{~mm}$ has also been selected to view the incidence of lower repetition rate interference over the degradation of the DCS. Regarding the amplitude set in the ESD gun, it has been set to $+12 \mathrm{kV}$, however, as the dielectric breakdown voltage is lower due to the proximity between the electrode and the metal, this tension is not reached.

\section{MEASUREMENT OF THE INTERFERENCES}

A detailed description of the instrumentation and the parameters configured to obtain the IQ components of the impulsive noise is explained in this section. Moreover, the time-domain results obtained for each of the radiated transient produced by the sparks are also shown.

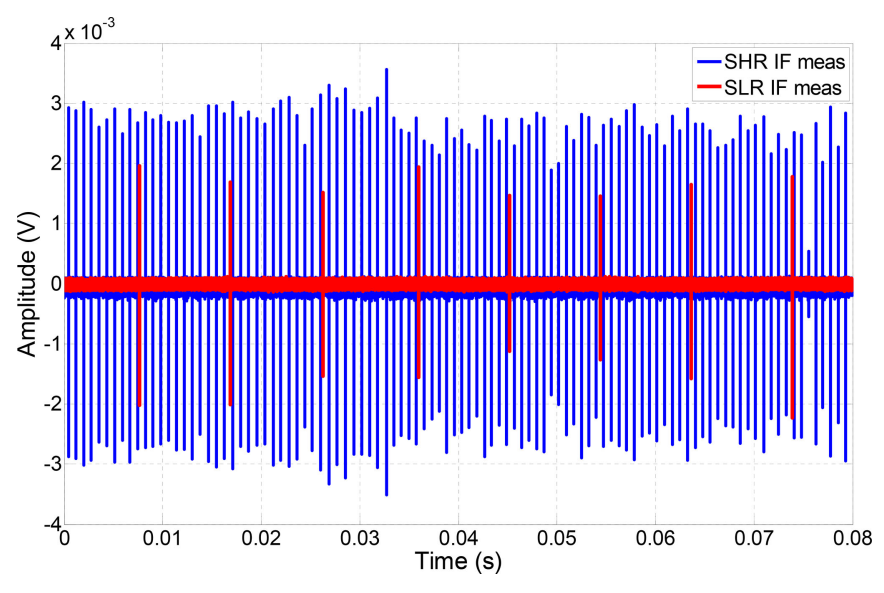

Fig. 2. Time-domain measurement of the IF signal when the SLR and SHR interferences are produced.

As it has been mentioned earlier, two different radiated transient interferences have been generated to try to produce disturbances to the GSM downlink. The downlink is the problematic link in the GSM-R system because the signal level received from the BTS is extremely weak. When the electrode of the ESD generator is at minimum distance to the metal, sparks with higher repetition rate are produced; we have named this interference as sparks high rate (SHR) interference. Otherwise, when the metal is placed at a distance of $1 \mathrm{~mm}$, sparks with lower repetition rate are generated, and the radiated transient has been named as sparks low rate (SLR) interference

For the time-domain acquisition, the GSM antenna is connected to the R\&S ESPI3 EMI receiver. The EMI receiver is centered in the frequency band of the downlink GSM channel 20, which corresponds to $939 \mathrm{MHz}$. Regarding the RBW employed in the EMI receiver, it was set to $3 \mathrm{MHz}$ for the SHR, while it was set to $10 \mathrm{MHz}$ for the SLR. The RBW setting shall be adjusted in order to provide sufficient amplitude level to the IF signal for improving the transient pulse identification and the overall sensitivity. The reference level of the EMI receiver is also configured to maximize the dynamic range without saturating the instrument. The IF output signal is used as the input of the Tektronix DPO 7104 oscilloscope, which is sampling at $125 \mathrm{MSamples} / \mathrm{s}$ as the IF output of the EMI receiver is 20.4 MHz. The total record length set to carry out the measurements is $10^{7}$ samples as it has been sufficient to measure all the main parameters of the transient interference. These 10 millions of samples correspond to a total acquisition time of $80 \mathrm{~ms}$.

Fig. 2 shows the IF signal captured by the oscilloscope when the SHR and SLR interferences are produced. The total time observed is equal to $80 \mathrm{~ms}$, where 104 pulses appear due to the high rate of the sparks produced by the breakdown of the air dielectric when the SHR interference is applied. Otherwise, when the SLR interference is measured, only eight pulses are acquired.

Once the IF measurement has been carried out, then, data are postprocessed to obtain the IQ components of each type of interference. Furthermore, in this stage, the interference 


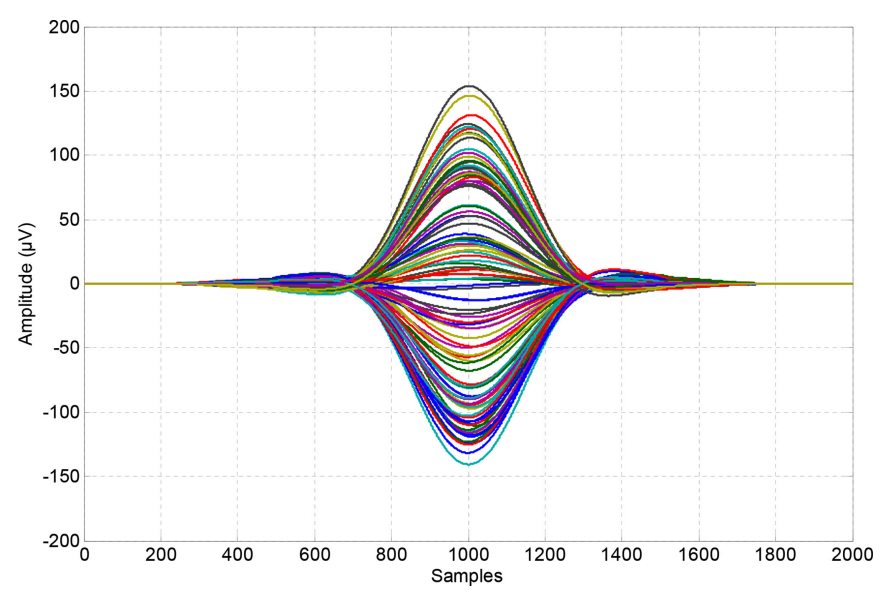

Fig. 3. In-phase component of the transient interference when the SHR interference is produced.

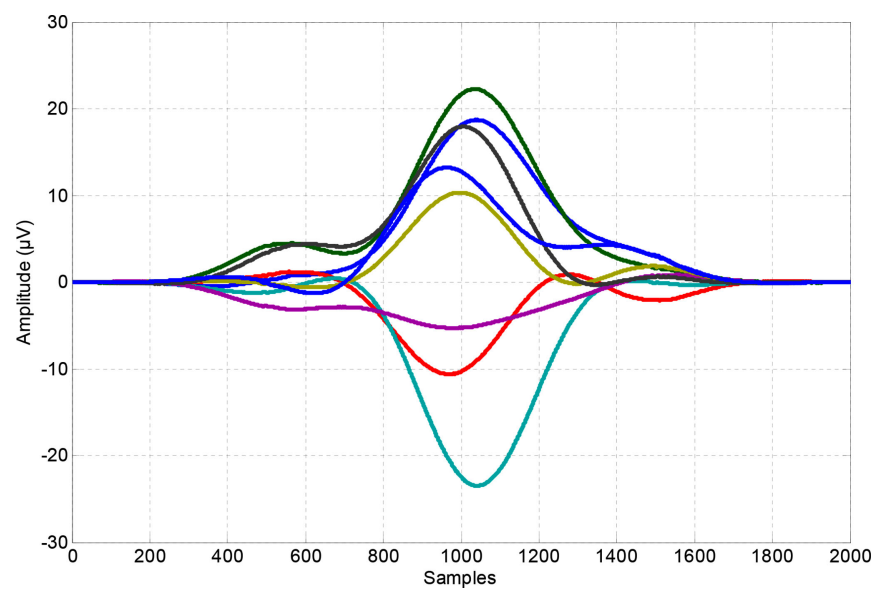

Fig. 4. In-phase component of the transient interference when the SLR interference is produced.

is bandpass filtered by the GSM frequency bandwidth in order to know the exact interference that would be received by the MS.

In Fig. 3, the in-phase component of the 104 transient pulses detected is represented after it has been filtered through $200-\mathrm{kHz}$ bandwidth, and in Fig. 4, the in-phase component of the SLR interference is represented overlapping the transient pulses that occurs in $80 \mathrm{~ms}$. The maximum value measured for the SHR interference is around $150 \mu \mathrm{V}$.

Regarding the SLR measurements, in comparison with the IQ components of the SHR interference, the amplitude has been reduced to $30 \mu \mathrm{V}$ and the number of pulses generated by the sparks has been reduced to only eight pulses in $80 \mathrm{~ms}$. Consequently, the repetition rate of the interference has been varied from a mean frequency of $1.3 \mathrm{kHz}$, when the SHR interference is generated to $100 \mathrm{~Hz}$ when the SLR interference is applied.

\section{Evaluation of the Degradation Produced Over THE GSM SYSTEM}

In this section, the results of both new procedures to determine the distortion produced by the two radiated transient in-
TABLE II

GSM TECHNICAL SPECIFICATION TO QUANTIFY THE SigNAL QUALITY

\begin{tabular}{lcc}
\hline \hline Quality Band & Range of actual BER & Assumed value \\
\hline RXQUAL_0 & Less than $0.2 \%$ & $0.14 \%$ \\
RXQUAL_1 & $0.2 \%$ to $0.4 \%$ & $0.28 \%$ \\
RXQUAL_2 & $0.4 \%$ to $0.8 \%$ & $0.57 \%$ \\
RXQUAL_3 & $0.8 \%$ to $1.6 \%$ & $1.13 \%$ \\
RXQUAL_4 & $1.6 \%$ to $3.2 \%$ & $2.26 \%$ \\
RXQUAL_5 & $3.2 \%$ to $6.4 \%$ & $4.53 \%$ \\
RXQUAL_6 & $6.4 \%$ to $12.8 \%$ & $9.05 \%$ \\
RXQUAL_7 & Greater than $12.8 \%$ & $18.1 \%$ \\
\hline \hline
\end{tabular}

terferences over the GSM system are compared with the mobile reference measurement.

\section{A. GSM Reference Equipment Measurement}

First, a direct evaluation procedure is used as the reference to determine if the developed methodologies results are in accordance. As it has been mentioned earlier, the measurement carried out involves the following equipments: (a MS) and a BTS simulated by the Hewlett-Packard GSM MS Test unit model HP8922 M. The aim of the measurement is to evaluate the downlink of the GSM system which can be interfered by the sparks produced close to the MS.

The GSM technical specification ETSI GSM 05.08 establishes that the MS shall report the received signal quality. In this technical specification, it is specified how the received signal quality shall be measured by the MS in a manner that can be related to an equivalent average BER before channel decoding. The parameter that it is used to record the quality of the received signal is RXQUAL. The GSM technical specification defines eight levels of RXQUAL that must be mapped to the equivalent BER as detailed in Table II.

It is important to remark that the BER probabilities values used to define a quality band are the estimated error probabilities before channel decoding. After channel decoding, the RXQUAL probability values are also specified but are different from the values described in Table I. In our application, the RXQUAL value before the decoding is provided by the MS and it is shown at the screen of GSM MS Test unit. In addition to the RXQUAL reported by the MS, BER measurement can be also carried out using the GSM MS Test unit providing more accurate measurements than the RXQUAL.

Once the quality parameters to quantify the degradation of the GSM communication system have been defined, the procedure to measure the influence of the radiated transient is explained as follows. In order to carry out the measurements, the link between the MS and the GSM MS Test unit is created. The GSM communication channel designated has been chosen arbitrarily without any specific precondition; the channel selected is number 20 which corresponds to an uplink frequency of $894 \mathrm{MHz}$ and the downlink frequency at $939 \mathrm{MHz}$. Regarding the output an input level set, the GSM MS Test unit has been configured providing the mobile a signal corresponding to an RXLevel equal to 25 , which means that the signal level is between -86 and 
$-85 \mathrm{dBm}$. If a lower level of the receiver signal is selected, it will imply that it will be easier to disturb the communication; on the other hand, if the received signal level is higher, it will be more difficult to interfere the communication system. Therefore, the level of signal received has been selected in terms of a reliable situation, considering that usually the GSM$\mathrm{R}$ communication system receives levels that vary from -40 to $-100 \mathrm{dBm}$.

Regarding the measurement provided by the equipment, the MS reports the RXQUAL level and the GSM MS Test unit performs a BER measurement of 10140 bits for each of the transient radiated interferences. When SHR interference is applied in the proximity of the MS, the RXQUAL reported by the MS is RXQUAL_4, meaning that the BER reported by the mobile is between $1.6 \%$ and $3.2 \%$. Additionally, the measurement carried out by the GSM MS Test unit reports a BER of $2.07 \%$ when 10140 bits are considered. When the interference with lower frequency is applied (SLR interference), the MS reports RXQUAL_0, which means that the BER is lower than $0.2 \%$. In addition, when the BER measurement is performed by the GSM MS Test unit, the BER measured is $0.01 \%$ when 10140 bits are considered.

From the results stated by the MS and the GSM MS Test unit, we observe that the influence of the radiated interferences generated by the sparks is worst when the interference with the highest spark rate is applied. When the interference with a mean rate of $100 \mathrm{~Hz}$ (SLR interference) is applied close to the MS, the degradation produced to the GSM system is negligible as the reported RXQUAL is zero. Otherwise, SHR interference produces a significant degradation to the quality of the GSM transmission, causing a BER around $2 \%$. Consequently, the interference with a mean repetition rate of $1.3 \mathrm{kHz}$ is capable to interfere meaningfully the GSM system.

\section{B. Results Employing IQ Interference Capture and GSM Base-Band Simulation}

The first developed methodology to evaluate the influence over the GSM system is based on the base-band simulation of the DCS, adding the IQ components previously measured.

Regarding the evaluation of the GSM system in the presence of the impulsive noise generated by the sparks, a simulation has been performed using Simulink. First, the baseband signal has to be modeled properly with the objective to reach accurate results. A GSM transmitter and a receiver are modeled according to the specifications defined in the GSM standards, also including the impulsive noise channel. Regarding the transmitter and receiver, they are divided into two main blocks; one block is responsible for generating the codification, while the other generates the modulation. In the coding block, the cyclic redundancy code is generated and also an interleaving of the bits is carried out. Afterward, in the modulation block, the Gaussian minimum shift keying (GMSK) modulation is produced as the GSM specification calls GMSK the physical layer modulation scheme. The main definition parameter of the GMSK modulation is the BT; where B is 3-dB bandwidth of the filter and $\mathrm{T}$ is the symbol duration. In the simulation, the bit rate selected is

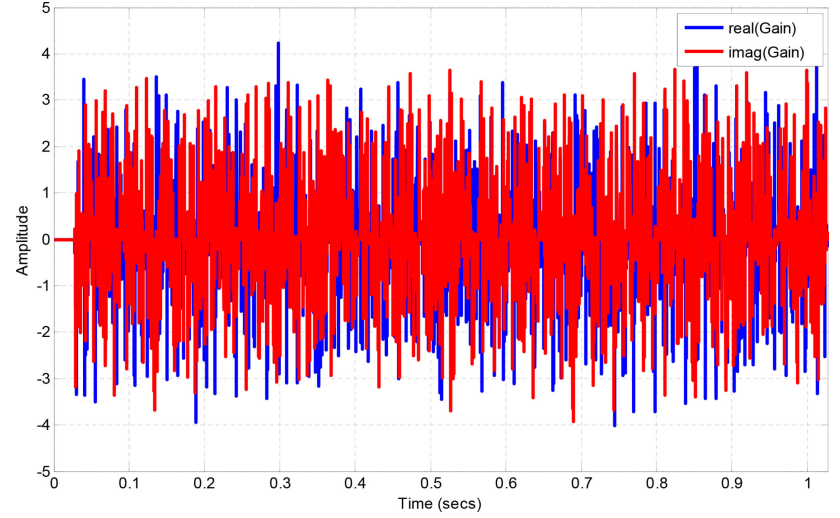

Fig. 5. IQ components of the SHR impulsive interference added to the baseband simulation after the amplitude normalization.

$9.6 \mathrm{kbps}$ and the BT parameter is assigned to 0.3. Concerning the bit-error measurement, the simulation permits us to obtain the BER before and after the coding is applied to the transmission. It is essential to consider that the results reported in the previous section by the GSM MS Test unit are provided before the decoding is produced; consequently, the BER computed before channel decoding will be the reference simulation data. In order to properly apply the interference in the simulated channel, the previously measured impulsive noise data must be normalized. A gain is included as the base band simulation is normalized to $1 \mathrm{~V}$. Therefore, if we want to evaluate the GSM system considering the received level by the MS, the interference must be multiplied by the factor to normalize the $-86 \mathrm{dBm}$ signal to $1 \mathrm{~V}$.

Regarding the simulated time, it has been fixed to $1 \mathrm{~s}$ in order to analyze a sufficient number of bits to achieve accurate results. During this period of time, the interference coming from the spark generator will be always present. Therefore, we are simulating the interference scenario where the transient interference longs at least $1 \mathrm{~s}$. However, the measurements presented in Section IV were performed only for $80 \mathrm{~ms}$ (see Fig. 2); consequently, a concatenation of the $80-\mathrm{ms}$ IQ measurements is done till the total time of $1 \mathrm{~s}$ is reached. This solution is the most optimized, because with the 80 -ms measurement, the main characteristics of the transient interference have been measured properly. Additionally, it is not necessary to store a full second sampled at $125 \mathrm{MSamples/s}$ which will have incurred in an unmanageable amount of data for the postprocessing.

Once the interference is added to the channel, the simulation can be run providing us the corresponding results when both interferences are taken into account. First, the high repetition spark interference case is simulated to analyze its influence over the GSM system. In Fig. 5, it is observed that the normalized IQ components are added to the channel when the SHR interference is applied and simulated. Regarding the BER produced by the interference, with the simulation, it is easy to estimate the errors produced as the transmitted bits are known. Consequently, when the SHR interference is present in the channel simulation, the BER obtained before decoding is $2.39 \%$, which corresponds to a RXQUAL_4.

Concerning the distortion produced by the SLR interference, a reduction of the degradation produced to the communication 


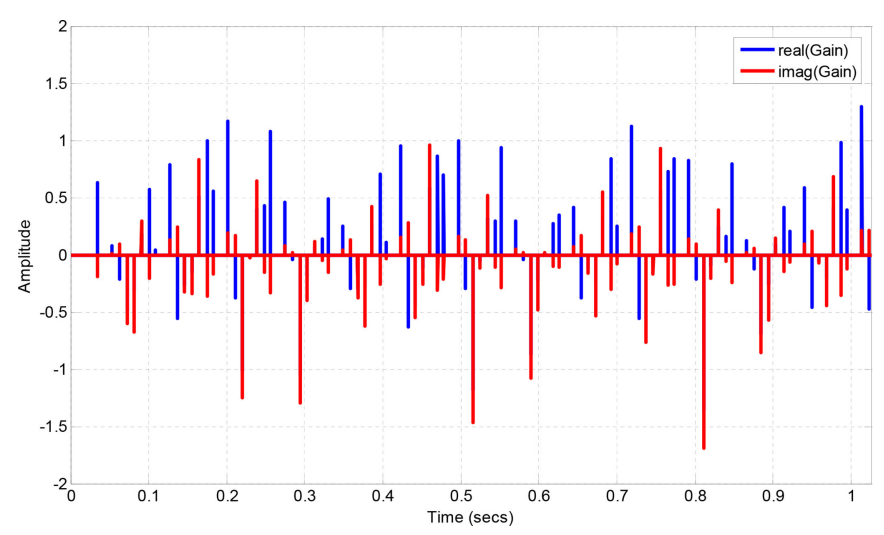

Fig. 6. IQ components of the SLR impulsive interference added to the baseband simulation after the amplitude normalization.

system in comparison with the SHR interference is expected. In Fig. 6, the IQ components of the SLR interferences added to the channel for the simulation are shown. If Fig. 6 is compared with Fig. 5, it is noticed that the distortion produced to the GSM system caused by the short-rate sparks interference will be reduced. Once the SLR interference simulation is carried out, the BER results before the decoding is $0.04 \%$, which is related to a RXQUAL_0, meaning that the interference produced to the GSM system is negligible.

\section{Results Employing the APD Diagram Including the GSM Specification Limits}

The second developed methodology to determine the degradation produced by the SHR and SLR interference uses the APD diagram. As it has been mentioned, limit points can be established to the APD diagram to know the degradation produced to the GSM communication system.

The IQ components, measured in Section IV for both interferences, are employed as the input to compute the APD diagram. The statistical properties of the radiated transient interferences are obtained in the downlink $939-\mathrm{MHz}$ channel of the GSM system and using the 200-kHz bandwidth. The results displayed for the rapid (SHR) and slow (SLR) interferences are shown in Fig. 7, as well as the computed case, when nonimpulsive interference is considered. Moreover, the limit points associated with each of the RXQUAL levels defined in the GSM specifications are also represented to rapidly identify the RXQUAL resulting level. The RXQUAL limit points which can be observed in Fig. 7 have been calculated according to (8). The level received by the mobile is $-86 \mathrm{dBm}$, and the corresponding modulation scheme for the GSM system is a QPSK, which means that the bits transmitted per symbol is $2(m=2)$ and $\beta=1$. Substituting the values in (8), the APD limits for each level of RXQUAL can be seen in Fig. 7. The resulting RXQUAL level will be determined considering the point below the line of the APD diagram. Additionally, if the APD diagram line is below all the limit points represented in the APD diagram, the RXQUAL value will be RXQUAL_0 which means a BER lower than $0.2 \%$.

From the results shown in Fig. 7, the APD curve for each of the transient interferences represents the exceeding probability

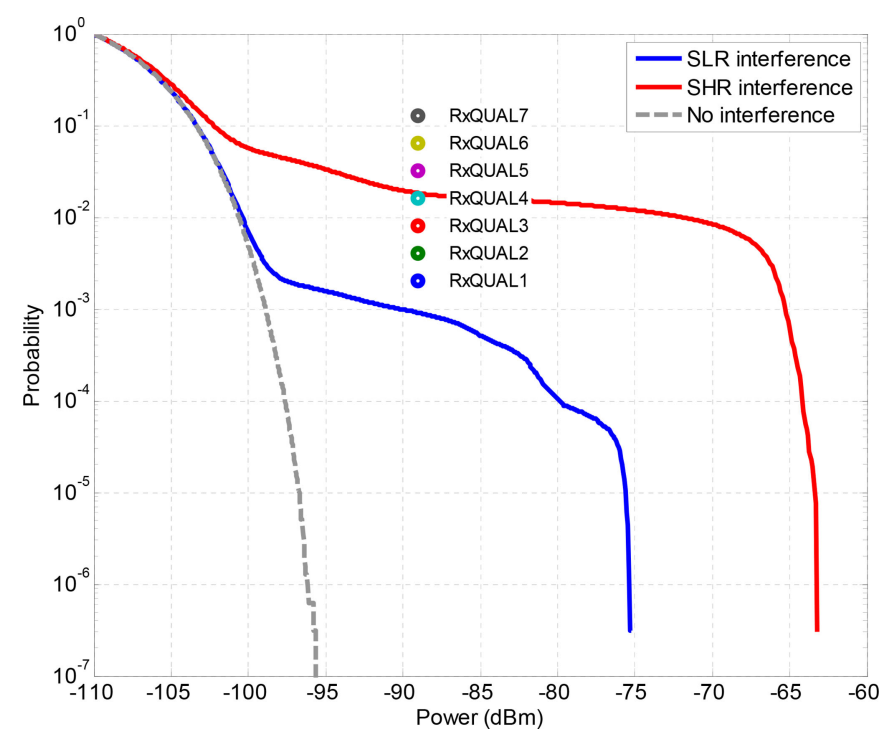

Fig. 7. APD diagram of the SHR and SLR interferences including the RXQUAL limits.

of the interference envelope amplitude. Therefore, it is easy to interpret the different slopes produced by each of the impulsive interferences. The SHR interference has higher amplitude and also higher probability than SLR interference, which means that in impulsive interferences such as the radiated transients, the repetition rate of the interference is also higher. Moreover, the limit points permit us to delimit that the SHR interference will produce a signal quality of RXQUAL_4 when the disturbance is present in the environment. On the other hand, from the APD diagram, it can also be concluded that the SLR interference will not cause any noticeable degradation to the GSM system, as the APD resulting curve is below all the RXQUAL levels. From the direct observation of the APD diagram, it can be established that the SHR produced a BER around $1.8 \%$. Otherwise, when the slow repetition spark interference is generated, the error rate is around $0.09 \%$.

The APD diagram has shown that the interference statistical information provided by the methodology is a useful and accurate tool to analyze the DCS performance rapidly and properly in the presence of radiated transient interferences. Considering the results shown in this section, the APD shows its powerful capabilities to be the best methodology to measure and analyze radiated transient interferences. Commonly, radiated transient interferences produce heavy-tailed distributions as it is observed in Fig. 7, this type of resulting distribution allows us to easily interpret the results from the APD diagram.

In addition, using the APD methodology with a single timedomain measurement, various GSM channels can be evaluated rapidly calculating the APD curve. As an example with the measurements performed in Section IV, where 3-MHz RBW was set in the EMI receiver, the APD diagram can be computed for either 15 GSM downlink channels. Otherwise, if a fullspectrum measurement is carried out employing time-domain methodologies developed [10], the APD diagram can be obtain in any channel of the GSM communication system. 
This article has been accepted for inclusion in a future issue of this journal. Content is final as presented, with the exception of pagination.

TABLE III

COMPARISON OF THE MEASURED-SimUlated DEGRADATION PRODUCED BY SHR AND SLR INTERFERENCES OVER THE GSM SYSTEM

\begin{tabular}{lccc}
\hline \hline Methodology & Interference & RXQUAL & BER \\
\hline Measured signal quality by MS & SHR & 4 & $2.07 \%$ \\
& SLR & 0 & $0.01 \%$ \\
DCS simulation & SHR & 4 & $2.39 \%$ \\
\multirow{2}{*}{ APD error estimation } & SLR & 0 & $0.04 \%$ \\
& SHR & 4 & $1.8 \%$ \\
& SLR & 0 & $0.09 \%$ \\
\hline \hline
\end{tabular}

\section{Methodologies COMPARISON AND CONCLUSIONS}

In this section, the results obtained following the different methodologies to evaluate the influence of both radiated transient interferences over the GSM downlink system are compared. The measurements reported by the GSM MS Test unit are the reference ones because an equipment transmitting information was placed under the radiated transient interferences. The measurement of the interferences and afterward performs the GSM simulation, and the APD diagram calculation are the methodologies developed to predict beforehand the degradation of the DCS. A summary chart of the results reached by the different methodologies is displayed in Table III.

Concerning the results obtained, first, it is essential to highlight the excellent agreement reached between all the different methodologies. When the impact of the SHR interferences is evaluated with the DCS simulation methodology and the APD error estimation, the RXQUAL_4 value anticipated is the same that is reported by the GSM MS Test unit. Additionally, if the BER differences are considered, the variation is around $0.3 \%$ compared with the reference value, which is a minimum difference if it is assumed that RXQUAL_4 level varies from 1.6\% to $3.2 \%$. Furthermore, if the results of the SLR interference are compared, each methodology concludes that a negligible interference effect is produced between the radiated transients and the downlink of the GSM system. All the methodologies associate the SLR interference to a received signal quality equal to RXQUAL_0, which incomes to an irrelevant interference scenario. Regarding the BER results related to the sparks generated around a repetition frequency of $100 \mathrm{~Hz}$ (SLR interference), all the results are below $0.1 \%$.

The results show that the developed methodologies have provided estimations of the BER with excellent accuracy when results are compared with the reference obtained from direct BER measurements by the GSM MS Test unit. Even if the results presented here cover a particular scenario, extensive measurement campaigns have shown that this methodology is sufficiently general and coherent to be reliably used to estimate the degradation of any DCS produced by a previously measured radiated transient interference. Beforehand, the IQ capture and DCS simulation procedure must be suitable to evaluate any communication system interfered by the transient noise if the system is properly modeled. Otherwise, the APD methodology can be employed when the DCS under evaluation are digital coherent radio receivers. Finally, it is necessary to emphasize the APD diagram practical usability, which has been especially valuable due to its straightforward quantification and interpretation of the degradation produced in the GSM system due to transient interferences.

\section{REFERENCES}

[1] D. Middleton, "Statistical-physical models of electromagnetic interference," IEEE Trans. Electromagn. Compat., vol. EMC-19, no. 3, pp. 106-127, Aug. 1977.

[2] M. Pous and F. Silva, "Prediction of the impact of transient disturbances in real-time digital wireless communication systems," IEEE Electromagn. Compat. Mag., vol. 3, no. 3, pp. 76-83, 3rd Quarter 2014.

[3] S. Dudoyer, V. Deniau, R.R. Adriano, M. N. B. Slimen, J. Rioult, B. Meyniel, and M. Berbineau, "Study of the susceptibility of the GSM-R communications face to the electromagnetic interferences of the rail environment," IEEE Trans. Electromagn. Compat., vol. 54, no. 3, pp. 667-676, Jun. 2012.

[4] V. Deniau, H. Fridhi, M. Heddebaut, J. Rioult, I. Adin, and J. Rodriguez, "Analysis and modelling of the EM interferences produced above a train associated to the contact between the catenary and the pantograph," in Proc. Int. Symp. Electromagn. Compat., Sep. 2-6, 2013, pp. 721-726.

[5] A. Knobloch and H. Garbe, "Critical review of converting spectral data into prospective bit error rates," in Proc. IEEE Int. Symp. Electromagn. Compat., 1Aug. 19-23, 2002, vol. 1, pp. 173-178.

[6] V. Deniau, S. Dudoyer, M. Heddebaut, A. Mariscotti, A. Marrese, N. Pasquino, "Test bench for the evaluation of GSM-R operation in the presence of electric arc interference," in Proc. Elect. Syst. Aircraft, Railway Ship Propulsion, Oct. 16-18, 2012, pp. 1-6.

[7] P. F. Stenumgaard, "On radiated emission limits for pulsed interference to protect modern digital wireless communication systems," IEEE Trans. Electromagn. Compat., vol. 49, no. 4, pp. 931-936, Nov. 2007.

[8] K. Wiklundh, "Relation between the amplitude probability distribution of an interfering signal and its impact on digital radio receivers," IEEE Trans. Electromagn. Compat., vol. 48, no. 3, pp. 537-544, Aug. 2006.

[9] Y. Matsumoto, "On the relation between the amplitude probability distribution of noise and bit error probability," IEEE Trans. Electromagn. Compat., vol. 49, no. 4, pp. 940-941, Nov. 2007.

[10] M. Pous and F. Silva, "Full-spectrum APD measurement of transient interferences in time domain," IEEE Trans. Electromagn. Compat., vol. 56, no. 6, pp. 1352-1360, Dec. 2014.

[11] Y. Matsumoto and K. Gotoh, "A method of defining emission limits including the gradient of an amplitude-probability-distribution curve," in Proc. Int. Symp. Electromagn. Compat., Sep. 1-4, 2014, pp. 895-900.

[12] C. Jeruchim, "Techniques for estimating the bit error rate in the simulation of digital communication systems," IEEE J. Sel. Areas Commun., vol. SAC-2, no. 1, pp. 153-170, Jan. 1984.

[13] FP7 European Project TREND: Test of rolling stock electromagnetic compatibility for cross-domain interoperability. (2014). [Online]. Available: www.trend-eu.org

[14] N. B. Slimen, V. Deniau, J. Rioult, S. Dudoyer, and S. Baranowski, "Statistical characterisation of the EM interferences acting on GSM-R antennas fixed above moving train," Eur. Phys. J. Appl. Phys., vol. 48, p. 21202-1-21202-7, 2009.

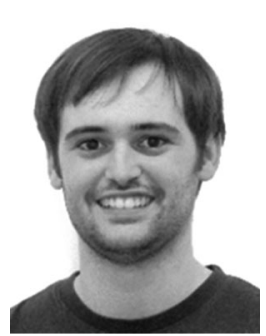

Marc Pous was born in Barcelona, Spain, in 1983. He received the M.Sc. degree in telecommunications engineering and the $\mathrm{Ph} . \mathrm{D}$. degree from the Universitat Politècnica de Catalunya (UPC), Barcelona, Spain, in 2009 and 2015, respectively.

From 2003 to 2006, he was with the Electromagnetic Compatibility Department, LGAI Technological Centre. In 2006, he joined the Electromagnetic Compatibility Group, UPC, where he has been participating in international and national research projects related with automotive, aerospace, railway, and medical industries. His research is focused on the development of time-domain measurement techniques to capture interferences which are not properly measured following the harmonized electromagnetic compatibility standards. He studied radiated transient interferences and digital communication systems evaluation for the Ph.D. degree. 


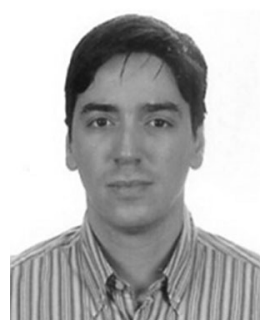

Marco A. Azpúrua (M'13) received the B.Sc. degree in telecommunications engineering and the M.Sc. degree in electrical engineering from the Universidad Central de Venezuela, Caracas, Venezuela, in 2008 and 2013, respectively. He is currently working toward the Ph.D. degree in the Electromagnetic Compatibility Group, Universitat Politècnica de Catalunya, Barcelona, Spain.

He was a Researcher in the Applied Electromagnetics Laboratory, Instituto de Ingeniería, Caracas. $\mathrm{He}$ is a member of the Venezuelan Standardization Committee for the Telecommunication Sector of CODELECTRA and FODENORCA and has participated in the adoption of the local standards of electromagnetic compatibility. His research interests include electromagnetic compatibility, antenna and microwave measurement technologies, and estimation of measurement uncertainty in complex systems and validation methods.

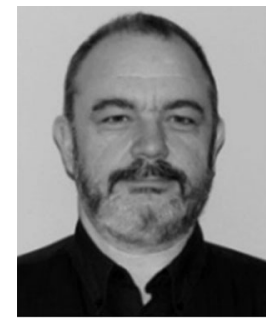

Ferran Silva (S'93-M'98) received the M.Sc. and Ph.D. degrees from the Universitat Politècnica de Catalunya (UPC), Barcelona, Spain, in 1989 and 1997, respectively.

He is currently an Associate Professor of electronics with the Department of Electronic Engineering, UPC. Since 2000, he has the Director of the Electromagnetic Compatibility Group, UPC, performing technology transfer activities to the industrial sector. His research interests include electromagnetic compatibility (EMC) in near field and time domain, including transients, with application to automotive, medical systems, and installations. He has contributed more than 90 papers to journals and conferences and contributed to the Wiley Encyclopedia of Biomedical Engineering. He has participated in 14 research projects related to EMC.

Dr. Silva is a member of the IEEE EMC Society and the Head of the Spanish chapter of this society; he is also member of the Spanish standardization committees SCTC77-210 and the CTN208 SCCISPR210A. He served as Chairman of the EMC Europe 2006 International Symposium. He belongs to the Board of Chairmen of EMC Europe. 